\title{
ON THE EXISTENCE OF AN INTEGRAL NORMAL BASIS GENERATED BY A UNIT IN PRIME EXTENSIONS OF RATIONAL NUMBERS
}

\author{
STANISLAV JAKUBEC, JURAJ KOSTRA, AND KAROL NEMOGA
}

\begin{abstract}
In the present paper a necessary condition for a cyclic extension of the rationals of prime degree $l$ to have an integral normal basis generated by a unit is given. For a fixed $l$, this condition implies that there exists at most a finite number of such fields. A computational method for verifying the existence of an integral normal basis generated by a unit is given. For $l=5$, all such fields are found.
\end{abstract}

Let the field $K$ be a Galois extension of the rational numbers of prime degree $l$. According to the Kronecker-Weber theorem there exists a positive integer $m$ such that $K \subset Q(m)$, where $Q(m)$ is the cyclotomic field of $m$ th roots of unity over $Q$. Let $m$ be the least such integer. In the field $K$ there exists an integral normal basis if and only if $m$ is squarefree (Leopoldt [1]).

In the present paper the existence of an integral normal basis generated by a unit in a prime extension of rational numbers $Q$ will be investigated. The procedure for solving this problem will be the following.

1. It is obvious that if an element generates an integral normal basis over $Q$, then its trace in $Q$ is \pm 1 . We will determine a necessary condition which a positive integer $m$ has to fulfill under the suppositions that $K \subset Q(m)$, and in the field $K$ there exists a unit $\varepsilon$ such that

$$
\operatorname{Tr}_{K / Q}(\varepsilon)= \pm 1
$$

We shall prove that for each prime $l$ there exists only a finite number of positive integers $m$ fulfilling this condition. So there is at most a finite number of fields $K$ of prime degree $l$ over $Q$ in which an integral normal basis is generated by a unit.

2. For each field $K$ of degree $l$ over $Q$ the problem of the existence of a normal basis generated by a unit will be solved. This solution will be computational and based on the isomorphism between a subgroup $E \subseteq Q(\omega)$, where $\omega=\sqrt[1]{1}$,

$$
E\{\gamma \in Q(\omega), N(\gamma)= \pm 1, \gamma \equiv \pm 1(\bmod 1-\omega)\},
$$

and the group $C_{l}$ of all circulant unimodular matrices of degree $l$.

Received June 12, 1989; revised December 7, 1989.

1980 Mathematics Subject Classification (1985 Revision). Primary 11R33. 
Let the field $K$ be an extension of $Q$ of prime degree $l$. Let there be an integral normal basis in the field $K$. Let $m$ be the least positive integer such that $K \subset Q(m)$. Since $m$ is squarefree, there exist distinct primes $p_{1}, p_{2}, \ldots, p_{s}$ such that

$$
m=p_{1} \cdot p_{2} \cdots p_{s} .
$$

Theorem 1. Let $\varepsilon$ be a unit in the field $K$ and $\operatorname{Tr}_{K / Q}(\varepsilon)= \pm 1$. Then

$$
l^{l} \equiv 1\left(\bmod p_{i}\right) \text { for all } i=1,2, \ldots, s,
$$

or

$$
l^{l} \equiv-1\left(\bmod p_{i}\right) \text { for all } i=1,2, \ldots, s,
$$

where the $p_{i}$ are the factors of $m$ given by (1).

Proof. By definition of $m$ it follows that $p_{i}$ is totally ramified in $K / Q$ for all $i=1,2, \ldots, s$. So, $\varepsilon \equiv a\left(\bmod \wp_{i}\right)$ for some rational integer $a$, where $\wp_{i}$ is the prime above $p_{i}$ in $K$. Therefore, $\pm 1=\operatorname{Tr}_{K / Q}(\varepsilon) \equiv l a\left(\bmod p_{i}\right)$ and $\pm 1=N_{K / Q}(\varepsilon) \equiv a^{l}\left(\bmod p_{i}\right)$. It follows that

$$
\operatorname{Tr}_{K / Q^{(\varepsilon)}}{ }^{l} \equiv l^{l} a^{l} \equiv N_{K / Q}(\varepsilon) l^{l}\left(\bmod p_{i}\right)
$$

for all $i=1,2, \ldots, s$. Therefore, either $l^{l} \equiv+1\left(\bmod p_{i}\right)$ for all $i$, or $l^{l} \equiv-1\left(\bmod p_{i}\right)$ for all $i$.

Our aim is to find all fields of given prime degree $l$ over $Q$ in which there exists an integral normal basis generated by a unit. If a unit $\varepsilon \in K$ generates an integral normal basis over $Q$, then $\operatorname{Tr}_{K / Q}(\varepsilon)= \pm 1$. Hence, by Theorem 1 , congruences (2) or congruences ( 3 ) hold. We shall give a computational method for verifying the existence of an integral normal basis generated by a unit.

For $l=2$, the solution of the problem is trivial. In the following, $l$ is an odd prime.

The field $K$ will be determined by the Galois group

$$
G=\Gamma(Q(m) / K) \subset(Z / m Z)^{*} .
$$

We need some further notation. For $i \in\{1,2, \ldots, s\}$, let $m_{i}=\frac{m}{p_{i}}$ and define the projection

$$
\operatorname{pr}_{i}: G \rightarrow\left(Z / m_{i} Z\right)^{*},
$$

where for $\sigma \in G, \operatorname{pr}_{i}(\sigma)$ is the restriction of $\sigma$ on $Q\left(m_{i}\right)$. By the symbol $H_{i}$ we denote the image $\operatorname{pr}_{i}(G)$.

Lemma 1. Let $L$ be the fixed field of the group $H_{i}$. Then $K \cap Q\left(m_{i}\right)=L$.

Proof. (a) First we show that if $x \in K \cap Q\left(m_{i}\right)$, then $x \in L$, i.e., $\psi(x)=x$ for all $\psi \in H_{i}$. Let $\psi \in H_{i}$. Then there exists $\psi^{\prime} \in G$ such that $\operatorname{pr}_{i}\left(\psi^{\prime}\right)=\psi$. Thus, $\psi(x)=\operatorname{pr}_{i}\left(\psi^{\prime}\right)(x)=x$. 
(b) Conversely, let $x \in L$. We have to prove that $x \in K \cap Q\left(m_{i}\right)$. Since $L \subset Q\left(m_{i}\right)$, it is sufficient to show that $x \in K$. Let $\psi^{\prime} \in G$. Then $\psi^{\prime}(x)=$ $\operatorname{pr}_{i}\left(\psi^{\prime}\right)(x)=x$. Hence $x \in K$.

Corollary 1. For $i=1,2, \ldots, s, H_{i}=\left(Z / m_{i} Z\right)^{*}$.

Proof. Since $[K: Q]=l$ is a prime, the field extension $K / Q$ has no nontrivial intermediary field.

Corollary 2. For all $p_{1}, p_{2}, \ldots, p_{s}$,

$$
p_{1} \equiv p_{2} \equiv \cdots \equiv p_{s} \equiv 1(\bmod l) \text {. }
$$

Proof. Let, for instance, $p_{s} \not \equiv 1(\bmod l)$. The homomorphism $\operatorname{pr}_{s}: G \rightarrow$ $\left(Z / m_{s} Z\right)^{*}$ is surjective by Corollary 1 . Hence,

$$
\left|\left(Z / m_{s} Z\right)^{*}\right||| G \mid
$$

It follows that

$$
\prod_{i=1}^{s-1}\left(p_{i}-1\right) \mid \frac{\prod_{i=1}^{s}\left(p_{i}-1\right)}{l},
$$

which contradicts $p_{s}-1 \not \equiv 0(\bmod l)$.

Example 1. Let $[K: Q]=5$. If in the field $K$ there exists a unit of trace 1 , then Theorem 1 determines all possible values of $m$ such that $K \subset Q(m)$ and $m$ is the least such positive integer. We have to find all primes $p, p \equiv 1(\bmod 5)$, fulfilling the congruences of Theorem 1,

$$
5^{5} \equiv 1(\bmod p) \text { or } 5^{5} \equiv-1(\bmod p) .
$$

We obtained the following four values of $m$ :

$$
m=11, \quad m=71, \quad m=521, \quad m=11 \cdot 71 \text {. }
$$

Let $\xi$ denote the primitive $m$ th root of unity. By $G$ we will denote the Galois group

$$
G=\Gamma(Q(m) / K) \subset(Z / m Z)^{*} .
$$

It is known that the numbers $\xi^{b}, b \in(Z / m Z)^{*}$, form an integral normal basis of the field $Q(m)$. So the following proposition holds.

Proposition 1. For a fixed $a \in(Z / m Z)^{*}-G$,

$$
\alpha_{1}=\sum_{x \in G} \xi^{x}, \alpha_{2}=\sum_{x \in G} \xi^{a x}, \ldots, \alpha_{l}=\sum_{x \in G} \xi^{a^{l-1} x}
$$

form an integral normal basis of the field $K$ over $Q$.

Let $C_{l}$ be the group of all unimodular circulant matrices of rank $l$. Let $\alpha_{1}, \alpha_{2}, \ldots, \alpha_{l}$ be an integral normal basis of the field $K$ defined by (4). Let $\beta_{1}, \beta_{2}, \ldots, \beta_{l}$ be an integral normal basis of the field $K$. Then we have

$$
\left(\beta_{1}, \beta_{2}, \ldots, \beta_{l}\right)=\left(\alpha_{1}, \alpha_{2}, \ldots, \alpha_{l}\right) \cdot A,
$$

where $A \in C_{l}$. 
We shall investigate the set of norms $\left\{N\left(\beta_{1}\right)\right\}$ for all integral normal bases $\left(\beta_{1}, \beta_{2}, \ldots, \beta_{l}\right)=\left(\alpha_{1}, \alpha_{2}, \ldots, \alpha_{l}\right) \cdot A, A \in C_{l}$, for a fixed prime modulus $p$.

Let $E$ be a subgroup of $Q(l)$,

$$
E=\{\gamma \in Q(l) ; \gamma \text { is a unit, } \gamma \equiv \pm 1(\bmod 1-\omega)\},
$$

where $\omega=\sqrt[i]{1}$. An element $\gamma \in E$ can be expressed in the form

$$
\gamma=b_{1} \omega+b_{2} \omega^{2}+\cdots+b_{l-1} \omega^{l-1} .
$$

Since $\gamma \equiv \pm 1(\bmod 1-\omega)$ and $\gamma \equiv-\operatorname{Tr}_{Q(l) / Q}(\gamma)(\bmod 1-\omega)$, the following congruence holds:

$$
b_{1}+b_{2}+\cdots+b_{l-1} \equiv \pm 1(\bmod l) \text {. }
$$

Hence, for $l \neq 2$, there exists a unique integer $c$ such that

$$
b_{1}+b_{2}+\cdots+b_{l-1}+l \cdot c= \pm 1 \text {. }
$$

We define a mapping $\Phi$, from $E$ into a set of circulant matrices of rank $l$ : for $\gamma \in E$, let

$$
\Phi(\gamma)=\operatorname{Circ}_{l}\left(a_{1}, a_{2}, \ldots, a_{l}\right),
$$

where $a_{1}=c, a_{2}=b_{1}+c, \ldots, a_{l}=b_{l-1}+c$.

Lemma 2. The mapping $\Phi$ is an isomorphism of groups $E$ and $C_{l}$.

Proof. Since the number $c$ is determined uniquely, the mapping $\Phi$ is correctly defined. $\Phi$ is clearly a homomorphism, i.e.,

$$
\boldsymbol{\Phi}\left(\gamma_{1} \cdot \gamma_{2}\right)=\boldsymbol{\Phi}\left(\gamma_{1}\right) \cdot \boldsymbol{\Phi}\left(\gamma_{2}\right)
$$

for all $\gamma_{1}, \gamma_{2} \in E$. The formula for a determinant of a circulant matrix, $\operatorname{det} \operatorname{Circ}_{l}\left(a_{1}, a_{2}, \ldots, a_{l}\right)=\left(a_{1}+\cdots+a_{l}\right) \cdot N_{Q(l) / Q}\left(a_{1}+a_{2} \omega+\cdots+a_{l} \omega^{l-1}\right)$, implies that $\Phi$ is into the group $C_{l}$. Directly from the definition of $\Phi$, it follows that $\Phi$ is a surjection and an injection.

The group $E$ is a subgroup of the group of all units of the field $Q(l)$. Let $t=(l-1) / 2-1$ and $\eta_{1}^{\prime}, \eta_{2}^{\prime}, \ldots, \eta_{t}^{\prime}$ be fundamental units of the field $Q(l)$. Clearly, there is a positive integer $a$ such that

$$
\left(\eta_{1}^{\prime}\right)^{a} \in E,\left(\eta_{2}^{\prime}\right)^{a} \in E, \ldots,\left(\eta_{t}^{\prime}\right)^{a} \in E .
$$

And so there exist $t$ fundamental units $\eta_{1}, \eta_{2}, \ldots, \eta_{t}$ of the group $E$. (Every finitely generated torsionfree module has a basis.) Hence, for any $\gamma \in E$, we have

$$
\gamma=(-\omega)^{n} \cdot \eta_{1}^{c_{1}} \cdot \eta_{2}^{c_{2}} \cdots \eta_{t}^{c_{t}}, \quad n, c_{1}, c_{2}, \ldots, c_{t} \in Z .
$$

Let $p \equiv 1(\bmod l)$. Let $Z(\omega)$ be the ring of integers of the field $Q(l)$. Let $\varepsilon$ be a unit of $Z(\omega)$. Hence, $(\varepsilon, p)=1$ in $Z(\omega)$ and $\varepsilon=b_{1} \omega+b_{2} \omega^{2}+\cdots+$ $b_{l-1} \omega^{l-1}$. The following congruence holds:

$$
\begin{aligned}
\varepsilon^{p} & =\left(b_{1} \omega+b_{2} \omega^{2}+\cdots+b_{l-1} \omega^{l-1}\right)^{p} \\
& \equiv b_{1}^{p} \omega^{p}+b_{2}^{p} \omega^{2 p}+\cdots+b_{l-1}^{p} \omega^{p(l-1)} \equiv \varepsilon(\bmod p) .
\end{aligned}
$$


Denote by $d$ the least positive integer such that $\varepsilon^{d} \equiv 1(\bmod p)$. Hence, $d \mid(p-1)$. The integer $d$ can be determined exactly.

Lemma 3. Let $\varepsilon=b_{1} \omega+b_{2} \omega^{2}+\cdots+b_{l-1} \omega^{l-1}$ be a unit of the ring $Z(\omega)$ and $f(x)=b_{1} x+b_{2} x^{2}+\cdots+b_{l-1} x^{l-1}$. Let $g$ be a primitive root modulo $p$ and $g_{1}=g^{(p-1) / l}$. Denote by $a_{k}, k=1,2, \ldots, l-1$, the least positive integer such that $f\left(g_{1}^{k}\right)^{a_{k}} \equiv 1(\bmod p)$. Then $d$ is the least common multiple of the numbers $a_{k}$.

Proof. For each $k=1,2, \ldots, l-1$ there exists exactly one prime divisor $\wp_{k}$ in $Z(\omega)$ such that $\wp_{k} \mid p$ and $\omega \equiv g_{1}^{k}\left(\bmod \wp_{k}\right)$. Therefore,

$$
\varepsilon=f(\omega) \equiv f\left(g_{1}^{k}\right)\left(\bmod \wp_{k}\right) .
$$

Since $1 \equiv \varepsilon^{d} \equiv f\left(g_{1}^{k}\right)^{d}\left(\bmod \wp_{k}\right)$, we have

$$
1 \equiv f\left(g_{1}^{k}\right)^{d}(\bmod p)
$$

for all $k=1,2, \ldots, l-1$.

Denote by the symbol $d^{*}$ the least common multiple of the numbers $a_{1}$, $a_{2}, \ldots, a_{l-1}$. From (5) we have $d^{*} \mid d$.

Since $\varepsilon^{d^{*}} \equiv 1\left(\bmod \wp_{k}\right)$ for all $k=1,2, \ldots, l-1$, we have that $\varepsilon^{d^{*}}-1$ is divisible by $p=\prod_{k=1}^{l-1} \wp_{k}$. Therefore, $\varepsilon^{d^{*}} \equiv 1(\bmod p)$ and $d \mid d^{*}$. This concludes the proof of Lemma 3.

We define matrices $A_{0}=\Phi(-\omega), A_{1}=\Phi\left(\eta_{1}\right), A_{2}=\Phi\left(\eta_{2}\right), \ldots, A_{t}=\Phi\left(\eta_{t}\right)$. And so for $A \in C_{l}$, we have

$$
A=A_{0}^{n_{0}} \cdot A_{1}^{n_{1}} \cdots A_{t}^{n_{t}}, \quad n_{0}, n_{1}, \ldots, n_{t} \in Z .
$$

As was shown above, when the set

$$
\left\{N\left(\beta_{1}\right) ;\left(\beta_{1}, \beta_{2}, \ldots, \beta_{l}\right)=\left(\alpha_{1}, \alpha_{2}, \ldots, \alpha_{l}\right) \cdot A, A \in C_{l}\right\}
$$

is investigated modulo $p$, it is sufficient to investigate a finite set of norms $\left\{N\left(\beta_{1}\right) ;\left(\beta_{1}, \ldots, \beta_{l}\right)=\left(\alpha_{1}, \ldots, \alpha_{l}\right) \cdot A\right\}, A=A_{0}^{n_{0}} \cdot A_{1}^{n_{1}} \cdots A_{t}^{n_{t}}, n_{0} \leq 2 l$, $n_{1} \leq d_{1}, \ldots, n_{t} \leq d_{t}$, where $d_{1}, \ldots, d_{t}$ are corresponding periods computable by Lemma 3 .

Example 2. In this example, all fields $K$ of degree 5 over $Q$ in which an integral normal basis generated by a unit exists will be determined.

Let $K$ be such a field. Then by Example $1, K \subset Q(m)$, where
(a) $m=11$,
(b) $m=71$,
(c) $m=521$,
(d) $m=11 \cdot 71$.

(a) $m=11$. The element $\alpha$ defined by (4) is a unit. Hence, the field $K \subset Q(11)$ of degree 5 over $Q$ has an integral normal basis generated by the unit $\alpha$.

(b) $m=71$. This is the same case as in part (a). $K \subset Q(71), K$ is of degree 5 over $Q$ and has an integral normal basis generated by the unit $\alpha$. 
(c) $m=521$. In this case, the element $\alpha$ has the norm $N_{K / Q}(\alpha)=-2083$. In the field $Q(5), t=(l-1) / 2-1=(5-1) / 2-1=1$. $(t$ is the number of fundamental units of the field $Q(5)$.) By computation it can be verified that $\eta_{1}=1+\omega^{3}-\omega^{4}$ is a fundamental unit of the group $E$. Put $A_{1}=\Phi\left(\eta_{1}\right)=$ $\operatorname{Circ}_{5}(1,0,0,1,-1)$. Define the sequence of norms

$$
u_{n}=N_{K / Q}\left(\beta_{1}\right) \text {, }
$$

where $\left(\beta_{1}, \ldots, \beta_{5}\right)=\left(\alpha_{1}, \ldots, \alpha_{5}\right) \cdot A_{1}^{n}, n \in Z$.

Let $p=11$. By a direct computation we have that the period $d_{1}=10$. Thus, the sequence $u_{n}$ is periodic, with the period 10 modulo 11 . By means of a computer it has been found that the sequence $u_{n}$ assumes these values modulo 11:

$$
\left(u_{1}, \ldots, u_{10}\right)=(9,4,8,4,7,10,2,9,4,7) .
$$

Hence, the numbers $N_{K / Q}\left(\beta_{1}\right)$, where $\left(\beta_{1}, \ldots, \beta_{5}\right)=\left(\alpha_{1}, \ldots, \alpha_{5}\right) \cdot A, A \in$ $C_{l}$, assume the values $\pm 9, \pm 4, \ldots, \pm 7$. (Since $A=A_{0}^{a} \cdot A_{1}^{n}, n \in Z, A_{0}=$ $\operatorname{Circ}_{5}(0,-1,0,0,0)=\Phi(-\omega)$.)

It follows from above that $\beta_{1}$, for $\left(\beta_{1}, \ldots, \beta_{5}\right)=\left(\alpha_{1}, \ldots, \alpha_{5}\right) \cdot A^{a} \cdot A_{1}^{n}$, can be a unit if $n \equiv 6(\bmod 10)$.

Now the sequence $u_{n}$ will be investigated modulo 61 . In this case, the period $d_{1}=15$. Clearly, it is sufficient to investigate the numbers $u_{n}, n=1,6,11$. We have that $u_{1} \equiv 50, u_{6} \equiv 54, u_{11} \equiv 26(\bmod 61)$, which are all different from $\pm 1(\bmod 61)$.

Thus, in the field $K \subset Q(521),[K: Q]=5$, an integral normal basis generated by a unit does not exist.

(d) $m=11.71$. In the field $Q(11.71)$ there exist four subfields $K$ of degree 5 over $Q$, corresponding to subgroups of the group $(Z / 11 \cdot 71 Z)^{*}$ of index 5 , the projections of which are surjective:

1. the field $K_{1}$ corresponding to the subgroup generated by 122,717 ;

2. the field $K_{2}$ corresponding to the subgroup generated by 122,475 ;

3. the field $K_{3}$ corresponding to the subgroup generated by 122,343 ;

4. the field $K_{4}$ corresponding to the subgroup generated by 122,200 .

In all four cases $1-4$, the sequence $u_{n}$ modulo 61 was investigated. The following values were obtained:

\begin{tabular}{c|ccccccccccccccc}
$u_{i}$ & 1. & 2. & 3. & 4. & 5. & 6. & 7. & 8. & 9. & 10. & 11. & 12. & 13. & 14. & 15. \\
\hline$K_{1}$ & 20 & 14 & 36 & 60 & 20 & 0 & 19 & 24 & 42 & 21 & 40 & 52 & 44 & 36 & 17 \\
$K_{2}$ & 7 & 13 & 46 & 53 & 20 & 41 & 47 & 39 & 38 & 30 & 27 & 35 & 7 & 34 & 49 \\
$K_{3}$ & 23 & 51 & 43 & 41 & 14 & 36 & 41 & 52 & 55 & 25 & 57 & 21 & 59 & 3 & 11 \\
$K_{4}$ & 32 & 41 & 31 & 24 & 18 & 45 & 27 & 34 & 34 & 1 & 50 & 6 & 48 & 30 & 31
\end{tabular}

In case 1 , for $n=4$ we have $u_{n} \equiv-1(\bmod 61)$. Using computations modulo 31 , where the period of the sequence $u_{n}$ is $d_{1}=30$, the following 
values were obtained:

$$
u_{4} \equiv 14, \quad u_{19} \equiv 26(\bmod 31),
$$

different from \pm 1 .

In case 4 , for $n=10$ we have $u_{n} \equiv 1(\bmod 61)$. In the same manner,

$$
u_{10} \equiv 11, \quad u_{25} \equiv 11(\bmod 31) \text {. }
$$

It follows from above that in the field $K, K \subset Q(11 \cdot 71)$, of degree 5 over $Q$ an integral normal basis generated by a unit does not exist. $(11.71$ is the least number $m$ such that $K \subset Q(m)$.)

\section{ACKNOWLEDGMENT}

We would like to thank the referee for simplifying the proof of Theorem 1 .

\section{BiBLIOGRAPHY}

1. H.-W. Leopoldt, Zur Arithmetik in abelschen Zahlkörpern, J. Reine Angew. Math. 209 (1962), 54-71.

2. P. J. Davis, Circulant matrices, Wiley, New York, 1979.

Slovak Academy of Sciences, Institute of Mathematics, Śtefánikova 49, 81473 Bratislava, CZechoslovakia 